DOI: https://dx.doi.org/10.26808/rs.ca.i10v5.03 International Journal of Computer Application (2250-1797)

Issue 10 Volume 5, September- October 2020

\title{
A Low-Complexity Signal Addition Method for PAPR Reduction in OFDM Systems
}

Stephen Kiambi ${ }^{\# 1}$, Elijah Mwangi ${ }^{\# 2}$, George Kamucha ${ }^{\# 3}$
\#1 School of Engineering, University of Nairobi, Nairobi, Kenya,
email: skiambi@uonbi.ac.ke
\#2 School of Engineering, University of Nairobi, Nairobi, Kenya,
email: elijah.mwangi@ uonbi.ac.ke
\#3 School of Engineering, University of Nairobi, Nairobi, Kenya,
email: gkamucha@uonbi.ac.ke

\section{ABSTRACT}

OFDM remains the transmission technique of choice for communication systems requiring high-speed transmission of data. However, signals generated via OFDM can exhibit high PAPR; a problem that still needs a practical solution. This paper proposes a fast-converging and low-complexity signal addition method for reducing the PAPR. The resources for transmitting the peak-reducing signal are reserved in the time domain rather than in the frequency domain as is done in tone reservation methods. The method has computational complexity of $O(M)$, where $M$ is the number of nonzero samples in the peak-reducing signal. Therefore, the overall computational complexity of the transmitter is barely increased because $O(M)$ is far muchless than $O\left(N \log _{2} N\right)$ of the IFFT in the transmitter. The proposed method can achieve high PAPR reductions with only a small number of nonzero samples in the peakreducing signal without affecting the average power of the transmitted signal and at a small data rate loss. For example, with $M=5 \%$ of 256-point IFFT samples, a PAPR reduction of 5.64 $\mathrm{dBcan}$ be achievedwith data rate lossof $4.8 \%$.

Key words:High Power Amplifier (HPA), Orthogonal Frequency Division Multiplexing (OFDM),Peak-to-Average Power Ratio (PAPR), Tone Reservation (TR).

Corresponding Author:Stephen Kiambi

\section{INTRODUCTION}

Orthogonal Frequency Division Multiplexing (OFDM) is a multicarrier transmission technique that employs parallel subcarriers to achieve high data rate transmission. The subcarriers are mutually orthogonal and the transmission system has high spectral efficiency. In addition, the system has symbol duration and a guard interval that are much longer than maximum multipath delay in order to eliminate both inter-symbol and inter-carrier interferences, and therefore allow use of a simple single-tap equalizer at the receiver to recover signals. These advantages, among others, have made OFDM the established transmission technique in high data rate applications such as digital audio broadcasting (DAB), digital video broadcasting (DVB),IEEE 802.11 WLAN and IEEE $802.16 \mathrm{a}$ WMAN standards, $4^{\text {th }}$ generationand $5^{\text {th }}$ generation mobile communication networks[1].

However, owing to the summation of many modulated subcarrier signals, an OFDM signal may suffer an unacceptably high peak-to-average power ratio (PAPR) especially when the number 
DOI: https://dx.doi.org/10.26808/rs.ca.i10v5.03 International Journal of Computer Application (2250-1797) Issue 10 Volume 5, September- October 2020

of subcarriers is large. Distortionless processing of high PAPR signals requires the high power amplifier (HPA) in the transmitter to be input backed-off away from the 1-dB compression point to avoid nonlinear amplification effects, mainly; bit-error rate (BER)degradation and out-of-band radiations.

However, an input backed-off HPA has lower power efficiency and thus high power consumption. This has the negative effect of increasing the cost of the device and reducing the lifetime of battery power at user terminals[2]. A better solution then is to reduce the PAPR to suitable levels before processing of the OFDM signal in the HPA.

Recently, different methods for reducing PAPR have been proposed in literature. These include signal coding[3], clipping and companding[4], selective mapping[5], partial transmit sequence [6] and tone reservation[7],[8]. The aim has always been to develop practically realisable methods with high PAPR reductions and low computational complexities. The tone reservation (TR)method is the most promising signal addition method because it does not distort the user data and hence has minimum BER degradation. Additionally, it does not require transmission of any side information for the demodulation process at the receiver.

Several tone reservation methods have been proposed. In [9], a proposed tone reservation (CF-TR) method designs peak cancelling signals by curve-fitting them to clipping noise signals. However, the method has to evaluate the highly computational intensive Moore-Penrose generalised matrix inversion during each iteration, and the resulting peak-reduced signal has an increased average power. A least-squares approximation TR (LSA-TR) method was proposed in [10]. Although the method has fast convergence, the PAPR reduction performance is poor, and has high computational complexity and leads to increased average transmit power. In [11] a TR method based on artificial neural networks and initial value optimisation (IVO-TR) is proposed to reduce runtime computational complexity by pre-generating peak-cancelling signals. At runtime, the method classifies an OFDM symbol and then searches for an appropriate peak-cancelling signal stored in the pre-work table. However, the method requires a very long pre-work time in order to generate near optimal peak-cancelling signals, and still has high runtime complexity, and results to increased average transmit power.

In this paper, we propose a low-complexity signal addition method with fast convergence. The key idea is to use the desired peak-reducing signal, which is the signal above a predetermined clipping level in an OFDM signal. The method avoids reservation of tones and the high complexity of determining the dummy weights in tone reservation methods by extending the peak-reduced signal by a few samples of the peak-reducing signal. Compared with CF-TR, LSATR, and IVO-TR, the method achieves better PAPR reductions while minimally affecting the average power of OFDM signals. Due to the simplicity, reliability, and fast convergence of the method, it can easily be implemented in the OFDM transmitter section.

The rest of the paper is organized as follows: Section 2 defines PAPR and its measurement. Section 3outlines the general concept of the tone reservation technique. In Section 4 , the proposed method is presented. Section 5 provides simulation results and analysis, while conclusions are in Section 6.

\section{PAPR OF OFDM}

An OFDM signal is a sum of $N$ modulated subcarrier signals. At baseband level, over one symbol duration $T$, the continuous-time signal has the analytical equation:

$$
x(t)=\frac{1}{\sqrt{N}} \sum_{k=0}^{N-1} X(k) e^{j 2 \pi f_{k} t} .
$$

Here, $X(k)$ are the modulation symbols obtained from binary phase-shift keying or $M$-ary quadrature amplitude modulation. The subcarrier frequency $f_{k}$ is the product of the index $k$ and 
DOI: https://dx.doi.org/10.26808/rs.ca.i10v5.03 International Journal of Computer Application (2250-1797)

Issue 10 Volume 5, September- October 2020

the subcarrier spacing. The division by $\sqrt{N}$ ensures that the average power in the time and frequency domains are the same. Because the above formulation is quite similar to the standard inverse discrete Fourier transform (IDFT), the well-known fast Fourier transform (FFT) algorithm is used to implement OFDM signal processing.

Due to the formation in(1), an OFDM signal can have high envelope fluctuations that can lead to nonlinear processing in the HPA. The measurement of the extent of the high power fluctuations is given by the probability by which the maximum instantaneous power is above the linear range of the HPA. For this measurement, the PAPR of the signal is needed and is given by:

$$
\operatorname{PAPR}\{x(t)\}=\frac{\max \left\{|x(t)|^{2}\right\}}{\mathrm{E}\left\{|x(t)|^{2}\right\}}
$$

where, $E\left\{{ }^{\cdot}\right\}$ is the expectation operator.

Assuming a sufficiently large number of subcarriers and that the subcarrier signals are statistically independent, then by the central limit theorem, both the real and imaginary parts of $x(t)$ are Gaussian distributed. Consequently, the instantaneous magnitudes of $x(t)$ are Rayleigh distributed. This in turn means that $x(t)$ can have high PAPR or high amplitude well above the average value.

Because of the digital signal processing of OFDM signals, the continuous-time PAPR is usually estimated in the discrete-time as:

$$
\operatorname{PAPR}\{\boldsymbol{x}\}=\frac{\max _{0 \leq n \leq N-1}\left\{|x(n)|^{2}\right\}}{E\left\{|x(n)|^{2}\right\}} .
$$

where, the discrete-time signal $x=[x(0), x(1), \ldots, x(N-1)]^{T}$. For a close estimation of the PAPR,the sampling rate applied on $x(t)$ must be sufficiently higher than the Nyquist rate, typically by a factor $\geq 4$, in order to avoid skipping the peak value[12].

The level of PAPR is measured by the complementary cumulative distribution function (CCDF), which by definition is the probability that the PAPR is above a given threshold[13]

$$
\operatorname{Pr}\{\operatorname{PAPR}\{\boldsymbol{x}\}>\gamma\}=1-\left(1-e^{-\gamma}\right)^{N} .
$$

Here, $\gamma$ is a threshold value, $N$ is the total number of subcarriers, and $\operatorname{Pr}\{$.$\} is the probability$ operator.

From the CCDF formula, if the CCDF value on the left is fixed, then for a fixed $N$,a higher value of $\gamma$ indicates a higher PAPR and vice versa. This implies that the difference between two values of $\gamma$ at the same CCDF value can be used to measure the level of PAPR reduction and to judge how well a proposed method reduces PAPR.

\section{CONCEPT OF TONE RESERVATION}

It is the concept of reserving a smaller number of subcarriers, previously meant for carrying user data to carry a peak-reducing signal for a given OFDM signal. The reserved subcarriers are generally referred to as peak reduction tones (PRTs). Because of the reservation, the number of subcarriers for transmitting user data, and hence the data rate, is reduced. Both the peak-reducing signal and the OFDM signal are combined to give a low PAPR transmit signal. The tone reservation concept is illustrated in Fig. 1, where $X(k)$ and $C(k)$ are the modulation data symbols and peak-reducing weights, respectively. At the receiver, only the knowledge of the locations of the data-bearing subcarriers is necessary for the recovery of the user data. 


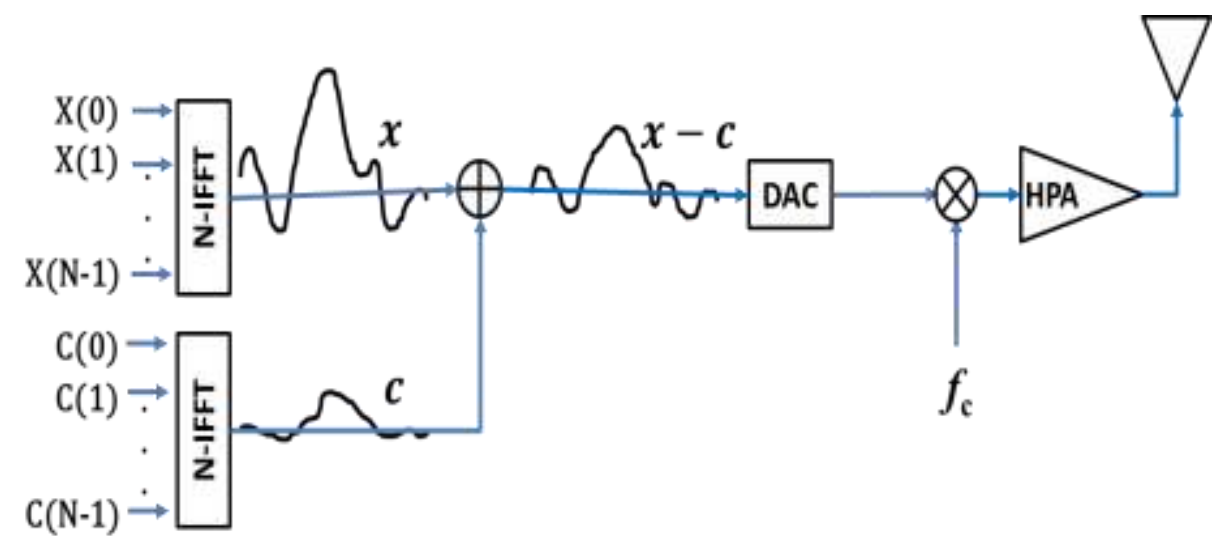

Fig.1. Tone reservation concept

In order to avoid any distortion on the user data, the data bearing vector and peakreducing weights must exist in two mutually exclusive frequency subspaces i.e. the data vector $\boldsymbol{X}=[X(0), X(1), \ldots, X(N-1)]^{T}$ should have all nonzero entries except in the reserved positions and likewise the peak-reducing vector $C=[C(0), C(1), \ldots, C(N-1)]^{T}$ should haveall zero entries except in the reserved positions. When the IDFT is applied to the two vectors, $\boldsymbol{X}$ and $\boldsymbol{C}$, it gives the discrete-time signals $x(n)$ and $c(n)$, which are, respectively, represented by $\boldsymbol{x}=[x(0), x(1), \ldots, x(N-1)]^{T}$ and $\boldsymbol{c}=[c(0), c(1), \ldots, c(N-1)]^{T}$. These two time-domain signals are added to give the transmit signals $(n)$ as follows:

$$
s(n)=x(n)-\frac{1}{\sqrt{N}} \sum_{k=0}^{N-1} C(k) e^{j 2 \pi k n / N}, \quad n=0,1, \ldots, N-1 .
$$

Equation (5)can be given in the matrix notation as:

$$
s=x-Q C
$$

where, $\boldsymbol{Q} \in \mathbb{C}^{N \times N}$ is the IDFT matrix with elements $(1 / \sqrt{N}) \exp (j 2 \pi k n / N)$, and all the vectors $\boldsymbol{x}, \boldsymbol{s}, \boldsymbol{C} \in \mathbb{C}^{N}$.If $L$ is the number of reserved subcarriers, then vector $\boldsymbol{C h a s} L$ nonzero elements, and the peak-reducing signal $\boldsymbol{c}=\boldsymbol{Q C}$, can be rewritten in the form:

$$
c=\widehat{Q} \widehat{C}
$$

where the new peak-reducing vector $\widehat{\boldsymbol{C}} \in \mathbb{C}^{L}$ contains only the nonzero elements of $\boldsymbol{C}$, and the submatrix $\widehat{\boldsymbol{Q}} \in \mathbb{C}^{N \times L}$ is made of only the $L$ columns of $\boldsymbol{Q}$ corresponding to the locations of reserved subcarriers.

The generation of the required peak-reducing signal requires finding the elements $\widehat{\boldsymbol{C}}$ that minimises the PAPR of the combined signal so that PAPR $\{\boldsymbol{s}\}<\operatorname{PAPR}\{\boldsymbol{x}\}$.To identify that there is reduction of the peak power in the peak-reduced signal compared to the original signal's average power, PAPR $\{\boldsymbol{s}\}$ is defined as follows:

$$
\operatorname{PAPR}\{\boldsymbol{s}\}=\frac{\max _{0 \leq n \leq N-1}\left\{|x(n)+c(n)|^{2}\right\}}{E\left\{|x(n)|^{2}\right\}} .
$$

The problem of finding $\widehat{\boldsymbol{C}}$ so thatPAPR $\{\boldsymbol{s}\}<\operatorname{PAPR}\{\boldsymbol{x}\}$ can be presented in the form of a minimax problem[14]

$$
\min _{\widehat{\boldsymbol{C}}} \max |\boldsymbol{x}-\widehat{\boldsymbol{Q}} \widehat{\boldsymbol{C}}|
$$


DOI: https://dx.doi.org/10.26808/rs.ca.i10v5.03 International Journal of Computer Application (2250-1797) Issue 10 Volume 5, September- October 2020

Where $\widehat{\boldsymbol{C}}$ is the optimization variable, and $\boldsymbol{x}$ and $\widehat{\boldsymbol{Q}}$ are the input parameters.

Solving the optimization problem in(9)is computationally intensive and can make implementation of the TR concept impractical especially in real-time systems. In addition, a system applying the concept may experience two major drawbacks. The first one is the increase in the average transmit power of the PAPR-reduced signal, and the second is the reduction in the data rate due to the use of PRTs that do not carry user data.

\section{PROPOSED METHOD}

This paper proposes a suboptimal PAPR reduction method that is derived from the general concept of tone reservations especially on the part of signal addition. The proposed method is referred to as"a low-complexity signal addition" method; in short LCSA method. Depending on the structure of the OFDM signal, and possibly the maximum allowed data rate loss, the method generates a desired peak-reducing signal (PRS), which it employs to reduce the PAPR. The reduction of PAPR is achieved by cancellation of all the highest peaks in the original OFDM signal. For exact cancellation of the highest peaks without introducing new ones, the ideal PRS should be composed of all the peaks above a threshold value called the clipping level. In addition, the PRS should have a phase spectrum identical to that of the OFDM signal.

Therefore, for a general complex discrete-time OFDM signal $x(n)$, the ideal PRScan be represented as a vector $\boldsymbol{d}=[d(0), d(1), \ldots, d(N-1)]^{T}$ whose elements are given by

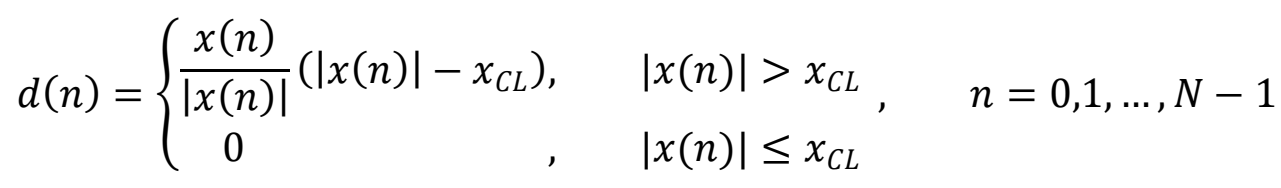

where $x_{C L}$ is the clipping level.

The aim of the proposed method is to create a PRS that matches the desired one in (10). However, to lower the computational complexity during the process of generating the PRS, the proposed method uses a simplified version of (10) that contains only the nonzero entries i.e.

$$
\widehat{\boldsymbol{d}}=[\hat{d}(0), \hat{d}(1), \ldots, \hat{d}(M-1)]^{T}
$$

where $M$ is the number of nonzero entries ind.

\subsection{LCSAAlgorithm}

From (7), and considering only the nonzero elements in $\boldsymbol{d}$,the design equation for the peakreducing signal is simply

$$
\widehat{c}=\widetilde{Q} \widehat{C}
$$

where $\widehat{\boldsymbol{c}} \in \mathbb{C}^{M}, \widehat{\boldsymbol{C}} \in \mathbb{C}^{L}$ and the new IFFT submatrix $\widetilde{\boldsymbol{Q}} \in \mathbb{C}^{M \times L}$ has only the rows from $\widehat{\boldsymbol{Q}} \in \mathbb{C}^{N \times L}$ corresponding to the Mindices of nonzero entries in $\boldsymbol{d}$. With $\hat{\boldsymbol{c}}$ calculated, the actual peak-reducing signal $\boldsymbol{c} \in \mathbb{C}^{N}$ is formed with all zero components except in $M$ positions whose values are those of vector $\hat{\boldsymbol{c}}$.

The task of designing a peak-reducing signal will then involve finding the elements of $\widehat{C}$ by solving the system of linear simultaneous equations given by:

$$
\widetilde{Q} \widehat{C}=\widehat{d}
$$

The solution to this system of equations is strongly dependent on the dimensions of the IFFT submatrix. Since $\widetilde{\boldsymbol{Q}}$ has Morthogonal rowsand $L$ orthogonal columns, the IFFT submatrix can have either a full row or column rank and thus there are two possible solutions. For both the square and underdetermined cases i.e. for $M \leq L$, the design will give an exact solution in the 
DOI: https://dx.doi.org/10.26808/rs.ca.i10v5.03 International Journal of Computer Application (2250-1797) Issue 10 Volume 5, September- October 2020

time domain i.e. $\widehat{\boldsymbol{c}}=\widehat{\boldsymbol{d}}$, while for the over determined case of $M>L, \hat{\boldsymbol{c}}$ will only approximate $\widehat{\boldsymbol{d}}$. The solution to the system of equations is therefore strongly dependent on the value of $M$ for a fixed value of $L$. The value of $M$ depends on the clipping that is applied to the OFDM signal.

For a given clipping level, $M$ can analytically be found from the distribution of the signal amplitudes. For this purpose, let $X_{n}$ denote a Rayleigh random variable representing the signal amplitudes whose cumulative distribution function is given by

$$
F_{X_{n}}(x)=1-e^{-\frac{x^{2}}{2 \sigma^{2}}}, \quad x \geq 0
$$

Where $\sigma$ is the scale parameter of the distribution. This distribution has a mean value given by the following equation:

$$
\bar{x}=\sigma \sqrt{\frac{\pi}{2}} .
$$

The clipping level can be defined as a function of the mean using

$$
x_{C L}=\lambda \bar{x}
$$

Where the clipping level parameter, $\lambda$, can have any value from zero to the maximum value of $\max (|x(n)|) / \bar{x}$.

From (14), the probability that some signal amplitudes are higher than the clipping level is given by:

$$
P\left(X_{n}>x_{C L}\right)=e^{-\frac{x_{C L}^{2}}{2 \sigma^{2}}}
$$

and since this is equal to $M / N$, then substituting(16)into (17)gives the number of nonzero entries in $\boldsymbol{d}$ as:

$$
M=N e^{-\frac{\pi}{4} \lambda^{2}}
$$

which is simply the number of peaks above the clipping level.

For the purposes of PAPR reduction, the clipping level should logically be set equal to or greater than the average value of the OFDM signal. From (18), the number of nonzero entries decreases exponentially with the clipping level parameter. The maximum value of $M$ occurs when the clipping level is equal to the average value which corresponds to $\lambda=1$, and $M=$ $0.4559 N$ orapproximately $46 \%$ of the total subcarriers. This value of $M$ is obviously much greater than the expected number of reserved subcarriers in any tone reservation method.

When $M$ is greater than $L$, the designed peak-reducing signal $\widehat{\boldsymbol{c}}=\widetilde{\boldsymbol{Q}} \widehat{\boldsymbol{C}}$ can only approximate the desired peak-reducing signal but can never be equal to it. The difference between the two signals is the residual error given by the equation:

$$
\widehat{\boldsymbol{\epsilon}}=\widetilde{\boldsymbol{Q}} \widehat{\boldsymbol{C}}-\widehat{\boldsymbol{d}}
$$

where the error vector $\hat{\boldsymbol{\epsilon}}=[\hat{\epsilon}(0), \hat{\epsilon}(1), \ldots, \hat{\epsilon}(M-1)]$ is composed of complex elements.

The main task of finding the elements of $\widehat{\boldsymbol{C}}$ can be done through least-squares minimisation[15], [16], [17]. The minimisation can yield a discrete-time signal that approximates or is equal to the desired peak-reducing signal. In the case $\widehat{\boldsymbol{c}}$ is not equal to $\widehat{\boldsymbol{d}}$, a good reduction of PAPR may not be achieved and there is also the possibility of having the average power of the PAPR-reduced signal increased. These two issues can be avoided by making the submatrix $\widetilde{\boldsymbol{Q}}$ squarei.e. to have $L=M$, and therefore obtain a unique optimal peak-reducing signal with the elements of $\widehat{\boldsymbol{C}}$ given by

$$
\widehat{\boldsymbol{C}}=\widetilde{\boldsymbol{Q}}^{-1} \widehat{\boldsymbol{d}}
$$


DOI: https://dx.doi.org/10.26808/rs.ca.i10v5.03 International Journal of Computer Application (2250-1797)

Issue 10 Volume 5, September- October 2020

There are only two possibilities of having $L=M$. One of the options is to fix $M$ and allow sufficient increase on the number of the reserved subcarriers but this has the undesirable effect of reducing the data rate of the system. The second, and the better, option is to fix $L$ and adjust $M$ in (18) using the clipping level parameter. For the different values of $M$, the optimal clipping level will be theoretically given by the equation:

$$
x_{C L}=\bar{x} \sqrt{\frac{4}{\pi} \ln \left(\frac{N}{M}\right)} .
$$

However, due to the random nature of the user data and the fixed number of reserved subcarriers, the theoretical clipping level in (21) may not always yield a value of $M$ that is equal to $L$ for all OFDM symbols. In addition, it is important to note that if the clipping level is increased to very high values, $M$ will be less than $L$ and thus making the system $\widetilde{\boldsymbol{Q}} \widehat{\boldsymbol{C}}=$ $\widehat{\boldsymbol{d}}$ underdetermined.Although for this case there are many exact peak-reducing signal solutions, only small PAPR reductions can be achieved. This is because the generated peak-reducing signal will have some few low amplitudes corresponding to a small number of the highest peaks in the OFDM signal.

Based on the above arguments, and depending on the target PAPR reduction, the clipping level can simply be set equal to one of the highest peak of the OFDM signal. This will ensure that we always have a square system of linear equations and therefore an optimal peak-reducing signal solution. For this setting, the peak-reduced signal is given by:

$$
\begin{aligned}
s & =\boldsymbol{x}-\boldsymbol{c} \\
& =\boldsymbol{x}-\boldsymbol{d}
\end{aligned}
$$

Because when $\boldsymbol{c}=\boldsymbol{d}$, all the high peaks will be cancelled without generating new ones, as found in methods that generate kernel-like peak-cancelling signals[18].

After fixing the clipping level, the system can also be allowed to utilise all the designated data subcarriers for user data transmission without reserving any for PAPR reduction. This is possible if the $M$ nonzero PRS samples are appended to the peak-reduced signal, just before the addition of cyclic prefix, for the recovery of the original OFDM signal at the receiver. The main blocks of the transmitter section of an OFDM system incorporating the PAPR reduction block are illustrated in Fig. 2. In the figure, $\boldsymbol{X}$ represents the modulation symbols while $\boldsymbol{x}, \boldsymbol{c}$, and $\boldsymbol{s}$ are the discrete-time OFDM signal, peak-reducing signal, and peak-reduced signal, respectively. The $\mathrm{CP}$ block represents the process of copying some samples from the tail end of the OFDM symbol to the front end for the creation of a guard interval.

The following is the summary of the proposedLCSAalgorithm.

\section{LCSA Algorithm}

i) Set total number of subcarriers $N$, data rate loss $R_{\text {loss }, t}$, and maximum allowed PAPR $\max$

ii) Generate OFDM signal $\boldsymbol{x}$, and calculate PAPR

iii) If PAPR $<\mathrm{PAPR}_{\max }$, transmit $\boldsymbol{x}$ and terminate the program, else got to step (iv)

iv) Set clipping level $x_{C L}$

v) Generate desired peak-reducing signald

vi) Calculate actual peak-reducing signalc

vii) Generate peak-reducing samples $\hat{\boldsymbol{c}}$

viii) Generate peak-reduced signal $\boldsymbol{s}=\boldsymbol{x}-\boldsymbol{c}$

ix) Append $\hat{\boldsymbol{c}}$ to $\boldsymbol{s}$ and pass the composite signal $\hat{\boldsymbol{s}}$ for onward processing

$\mathrm{x})$ End 
DOI: https://dx.doi.org/10.26808/rs.ca.i10v5.03 International Journal of Computer Application (2250-1797)

Issue 10 Volume 5, September- October 2020

At the receiver, after removing the cyclic prefix, the $M$ PRS samples are removed and added back to the peak-reduced signal to reconstruct the original OFDM time signal, which is then passed to the FFT block for demodulation.

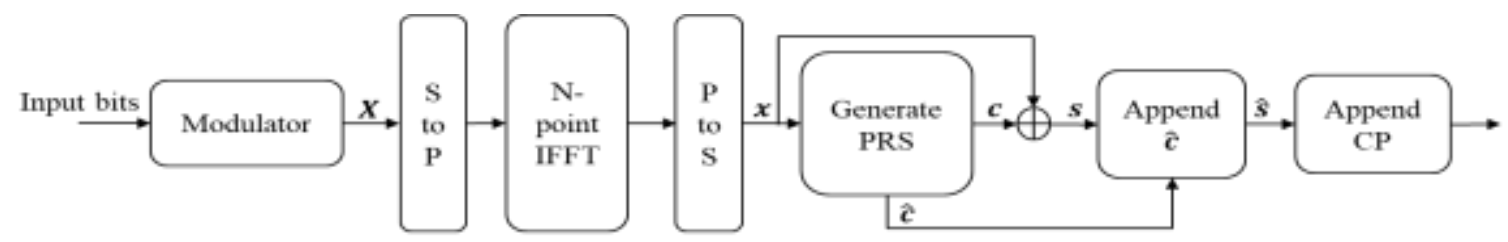

Fig.2. Transmitter with PAPR reduction block

Considering that in a typical implementation of the algorithm, $M$ will be much less than $N$, the waveform of the peak-reduced signal will be barely affected by the addition of $M$ samples. A typical composite signal for an OFDM system with $N=256$ and 5\% data rate loss is illustrated in Fig. 3 where the peak-reduced signal part is in blue and the peak-reducing signal part in red. As can be seen, the wave pattern generally remains that of the peak-reduced signal.

In the composite signal, all the user data is carriedin the peak-reduced signal part. Therefore, time-domain extension by $M$ samples will lead to a reduction in data rate by a factor of:

$$
R_{\text {loss }, t}=\frac{M}{N+M}
$$

The data rate loss in (23) is less than the one in frequency-domain implemented tone reservation methods, which due to the reserved subcarriers is given by:

$$
R_{\text {loss }, f}=\frac{M}{N} \text {. }
$$

In addition to the loss in (23), there will be a small reduction in the average power that also increases with the number of nonzero PRS samples. This can be explained by comparing the power in the clipped samples and the corresponding nonzero PRS samples to the power in the original samples. If we let $i$ be the index of a clipped sample, and $\hat{c}(i)$ and $x(i)$ be, respectively, the corresponding PRS and original signal samples, then the total power in the clipped and PRS samples will be less than or equal to the power in the original samples i.e.

$$
\sum_{i=1}^{M}\left(x_{C L}^{2}+\hat{c}^{2}(i)\right) \leq \sum_{i=1}^{M} x^{2}(i)
$$

Equation (25) implies that if $M$ increases, the average power reduction increases. However, since the sample amplitudes are between zero and one, the power reductions will be very small. Therefore, the power of the original OFDM signal will practically be retained.

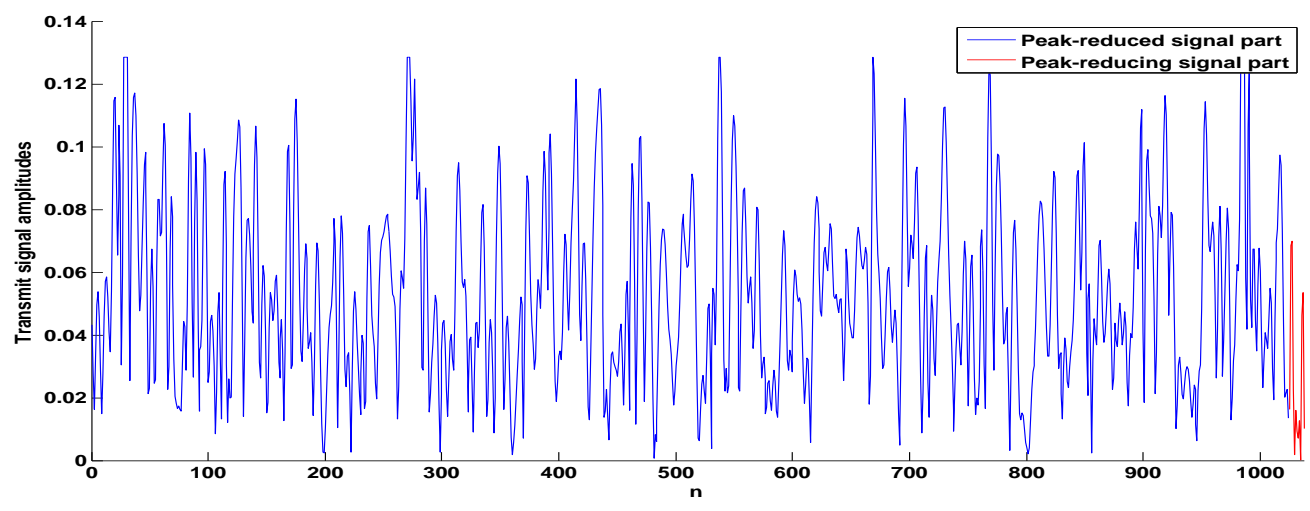


DOI: https://dx.doi.org/10.26808/rs.ca.i10v5.03 International Journal of Computer Application (2250-1797) Issue 10 Volume 5, September- October 2020

Fig.3. Composite transmit signal

\subsection{Computational Complexity}

The proposed LCSA algorithm has a very fast convergence rate because computing the required peak-reducing signal from the nonzero entries of the desired peak-reducing signal is done in a single step.

In general, the proposed algorithm has two main operations, which are the generation of peak-reducing signal using (10)and PAPR reduction through the addition of the OFDM signal and peak-reducing signal using (22). The generation of peak-reducing signal requires $2 M$ real multiplications and $M$ real additions while the operation of reducing PAPR requires $2 M$ real additions.

Therefore, the computational complexity of the proposed algorithm $O(M)$. But because $M \ll N$,the proposed algorithm will add insignificant computational complexity in OFDM systems in which it is deployed to reduce PAPR.

For comparison to IVO-TR, CF-TR and LSA-TR methods, their computational complexities are provided in Table 1. The IVO-TR has many pre-work computations during iteration process, training process and clustering. However, the iteration process, which uses the slow converging CC-TR[18]to generate peak reduction tones for training the self-organizing map of artificial neural networks, dominates the complexity. The algorithm's main parameters are the number of subcarriers $N$, number of samples $N_{s}$, number of iterations $I_{i v o}$, number of neurons in the input layer $N_{1}$ and number of neurons in the hidden layer $N_{2}$. The IVO-TR requires a long pre-work time in order to generate peak-reducing tones that are close to the ones generated by CC-TR. At runtime, the algorithm needs a process to recognise OFDM signal and table search for the right peak-cancelling signal.

For the CF-TR and LSA-TR, the number of iterations are, respectively, denoted by $I_{c f}$ and $I_{l s}$. These two algorithms start by calculating the clipping noise, which is the desired peak-cancelling signal. The LSA-TR then applies least square approximation on the noise to find the actual peak-cancelling signal. On the other hand, the CF-TR applies a generalised matrix inverse approximation on the noise. The two algorithms must in addition perform IFFT/FFT and other computations in each iteration and therefore, have a complexity that increases with the number of iterations.

In order to compare the runtime complexities, which is the most important for real-time systems, we first note that both $N_{1}$ and $N_{2}$ for the IVO-TR are typically greater than 100 . From the tabulated results, it can be observed that LCSA has in general a lower computational complexity and therefore, is more computationally efficient than the IVO-TR, CF-TR, and LSATR algorithms.

Table 1. Complexity analysis

\begin{tabular}{|c|c|c|c|c|}
\hline Algorithm & $\begin{array}{c}\text { Pre-work time } \\
\text { complexity }\end{array}$ & $\begin{array}{c}\text { Pre-work space } \\
\text { complexity }\end{array}$ & $\begin{array}{c}\text { Runtime } \\
\text { complexity }\end{array}$ & $\begin{array}{c}\text { Runtime space } \\
\text { complexity }\end{array}$ \\
\hline LCSA & None & None & $O(M)$ & $O(N)$ \\
\hline IVO-TR & $N_{s} \times I_{i v o} \times O\left(N \log _{2} N\right)$ & $N_{s} \times O(N)$ & $N_{2} \times O(N)$ & $\left(N_{1}+N_{2}\right) \times O(N)$ \\
\hline CF-TR & None & None & $I_{c f} \times O\left(N \log _{2} N\right)$ & $O(N)$ \\
\hline LSA-TR & None & None & $2 I_{l s} \times O\left(N \log _{2} N\right)$ & $O(N)$ \\
\hline & & & & \\
\hline
\end{tabular}




\section{RESULTS AND DISCUSSION}

The proposed LCSAmethodwasapplied to different OFDM systems, which were simulated in MATLAB. Systems under consideration, whose main parameters are listed in Table 2, were chosen for the purposes of ascertaining the method's performance and comparison with other promising PAPR reduction methods.

Table 2.Simulationinputs

\begin{tabular}{|c|c|}
\hline FFT size $N$ & 64,256 \\
\hline Subcarrier modulation & QPSK \\
\hline Number of OFDM symbols & $10^{5}$ \\
\hline Oversampling factor $F_{s}$ & 4 \\
\hline Data rate loss $R_{\text {loss }, \text { in } \%}$ & diverse \\
\hline Power amplifier model & Rapp model, $p=2, I B O=7 \mathrm{~dB}$ \\
\hline
\end{tabular}

As indicated in the table, during the simulations an oversampling factor, $F_{s}=4$, was applied to the discrete-time OFDM signal in order to closely estimate the continuous-time PAPR. All subcarriers were QPSK-modulated and this is sufficient for checking performance and comparing the proposed method with other methods because subcarrier modulation does not affect PAPR reductions. Both PAPR reduction and BER performances were analysed.

From(22), the generated PRS should match the desired signal and this is illustrated in Fig. 4. Without loss of generality, an OFDM system with 256 QPSK-modulated subcarriers was used to ascertain the PAPR reduction capability of the method. For this purpose, different low data rate losses, which are $R_{\text {loss }, t}=1.2 \%, 2.3 \%, 4.8 \%, 6.9 \%$, and $9.2 \%$ corresponding to $M=3,6,13,19$, and 26 nonzero PRS samples, respectively, were considered. The CCDF curves for the different values of $M$ are as shown in Fig. 5, and the PAPR reductions at $\mathrm{CCDF}=10^{-3}$ are tabulated in Table 3.

From the results in Table 3, it is evidentthat although the proposed LCSA method has a PAPR reduction capability that increases with the number of nonzero PRS samples, with only a few samples, high PAPR reductions can be achieved. Furthermore, the results of theaverage power increase show that the method approximately retains the average power of the original signal. For example with $M=26$, which seems quite high, the new average power is $95.3 \%$ of the original value before PAPR reduction.

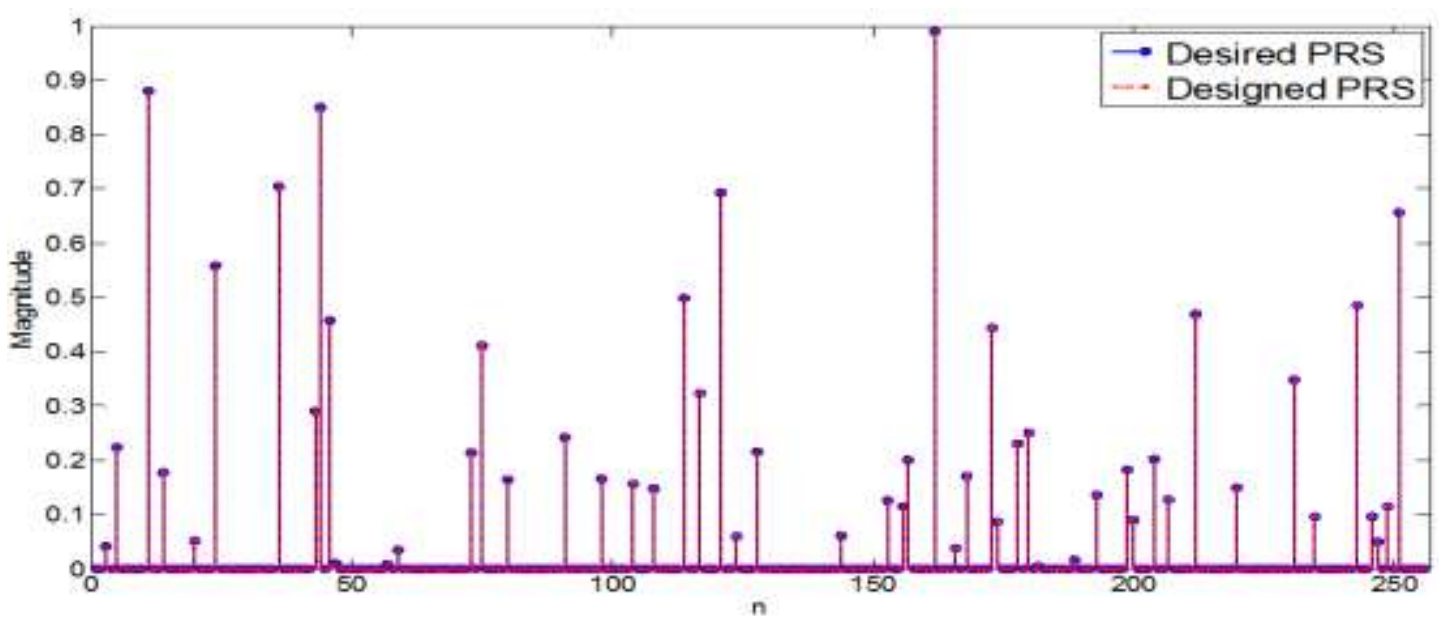

Fig.4. Desired and designed peak-reducing signals 
DOI: https://dx.doi.org/10.26808/rs.ca.i10v5.03 International Journal of Computer Application (2250-1797) Issue 10 Volume 5, September- October 2020

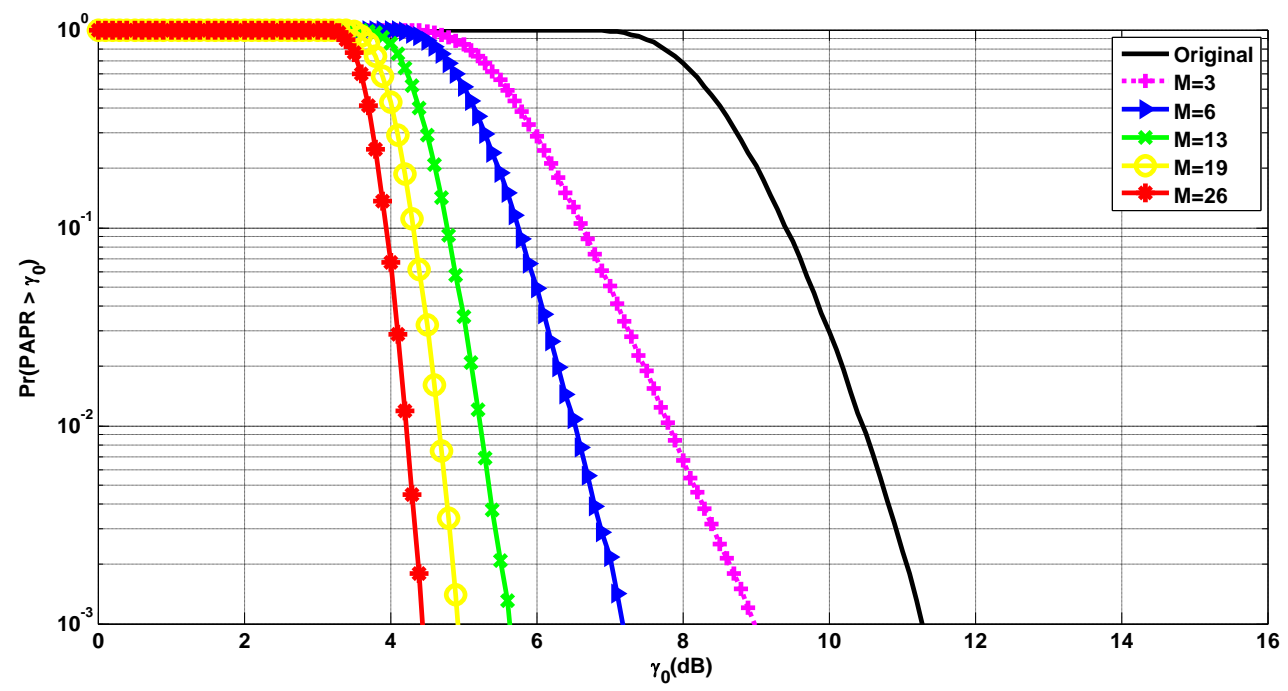

Fig.5. CCDFs for QPSK-modulated OFDM system with $N=256$

Table3. PAPR reductions for different number of PRS samples

\begin{tabular}{|l|l|l|l|l|l|}
\hline Number of PRS samples M & 3 & 6 & 13 & 19 & 26 \\
\hline Data loss rate $\boldsymbol{R}_{\text {loss }, \boldsymbol{t}}$ & $1.2 \%$ & $2.3 \%$ & $4.8 \%$ & $6.9 \%$ & $9.2 \%$ \\
\hline $\begin{array}{l}\text { PAPR Reduction in dB at } \\
\text { CCDF = 10 }\end{array}$ & 2.28 & 4.10 & 5.64 & 6.35 & 6.83 \\
\hline Average power increase in dB & -0.02 & -0.05 & -0.10 & -0.15 & -0.21 \\
\hline
\end{tabular}

After evaluating the accuracy of the proposed LCSA method, its performance was compared to CF-TR, IVO-TR, and LSA-TR methods. An OFDM system with $N=64$ subcarriers all QPSK-modulated, and of which $M=4$ are reserved, was used for evaluating the performance of each of the four systems. The simulation parameters for IVO-TR were $N_{1}=100$ neurons for the input layer, $N_{2}=200$ neurons for the hidden layer, and $10^{6}$ pre-work OFDM signals. The number of iterations was 3 for both the LSA-TR and CF-TR.

The CCDFs for the different methods are shown in Fig. 6 and the PAPR reductions at $\mathrm{CCDF}=10^{-3}$ are presented in Table 4 . As can be derived from the table, the proposed method has a PAPR reduction that is $0.6 \mathrm{~dB}, 1.0 \mathrm{~dB}$ and $1.9 \mathrm{~dB}$ higher than that of IVO-TR, CF-TR, and LSA-TR, respectively.

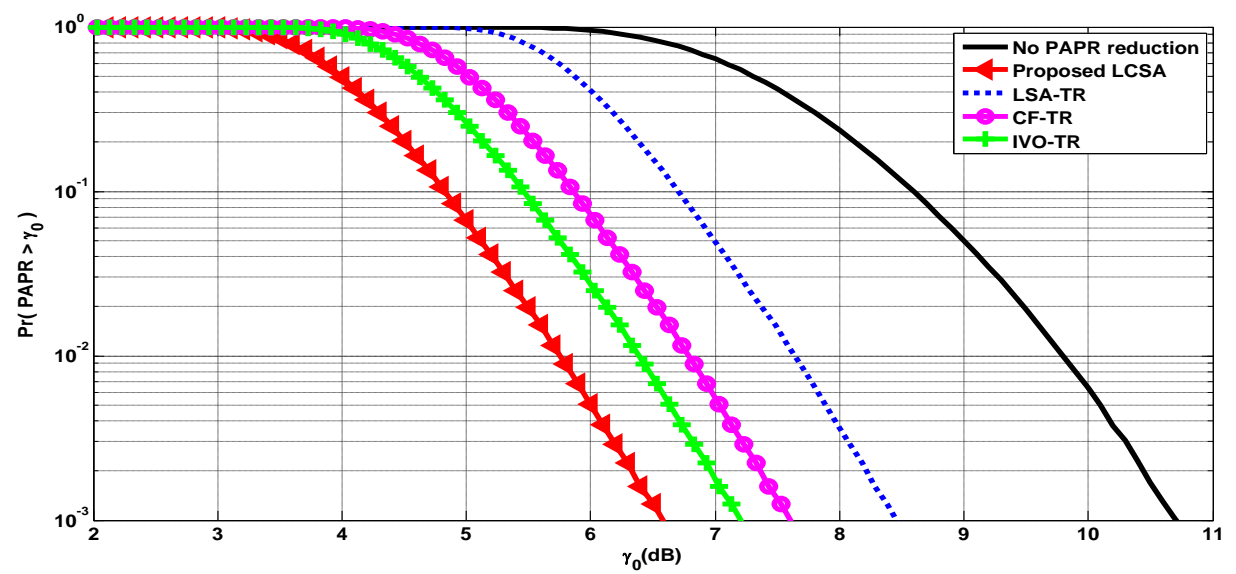

Fig.6. CCDFs for QPSK-modulated OFDM system with $\mathrm{N}=64$ and $\mathrm{M}=4$ 
DOI: https://dx.doi.org/10.26808/rs.ca.i10v5.03 International Journal of Computer Application (2250-1797) Issue 10 Volume 5, September- October 2020

Table4.PAPR reduction, average power increase, and data rate losses

\begin{tabular}{|c|c|c|c|}
\hline Algorithm & $\begin{array}{c}\text { PAPR } \\
\text { reduction in dB }\end{array}$ & $\begin{array}{c}\text { Average } \\
\text { power increase } \\
\text { in dB }\end{array}$ & Data rate loss \\
\hline LCSA & 4.1 & -0.12 & $5.88 \%$ \\
\hline IVO-TR & 3.5 & 0.57 & $6.25 \%$ \\
\hline CF-TR & 3.1 & 0.84 & $6.25 \%$ \\
\hline LSA-TR & 2.2 & 0.30 & $6.25 \%$ \\
\hline
\end{tabular}

Comparing the average power of the PAPR-reduced signal by the different methods, the results show that the proposed method is more power efficient than the rest because it has a power increase that is $0.42,0.69$, and $0.96 \mathrm{~dB}$ below that of the LSA-TR, IVO-TR, and CF-TR, respectively. On the data rate loss, the proposed method has the lowest loss of the four methods

Fig. 7 illustrates the simulated BER performances of the different methods. The Rapp model of the solid-state power amplifier (SSPA) was used with the smoothness parameter $p=2$. The input power back-off (IBO) was set to $7 \mathrm{~dB}$, which is slightly above the proposed method's PAPR of $6.56 \mathrm{~dB}$ at $\mathrm{CCDF}=10^{-3}$, in order to ensure that the probability of the clipped OFDM symbols was less than $1 \%$. After amplification through the HPA, the peak-reduced signal was sent over an additive white Gaussian noise (AWGN) channel.

In Fig. 7, the curve labelled "Theoretical" is the lower limit as it is the BER performance given by BER formula for the QPSK.The curve labelled "Without PAPR Red." is the worst caseupper limit as it gives the BER performance when the OFDM signal is passed through the HPA without first reducing the PAPR. The required bit energy to noise power spectral density, $E_{b} / N_{0}$, at BER $=10^{-5}$ of each method is presented in Table 5 . Based on the tabulated results, the proposed algorithm has the lowest BER degradation because the required $E_{b} / N_{0}$ is $0.02,0.05$, and $0.07 \mathrm{~dB}$ below that of the IVO-TR, CF-TR, and LSA-TR, respectively.

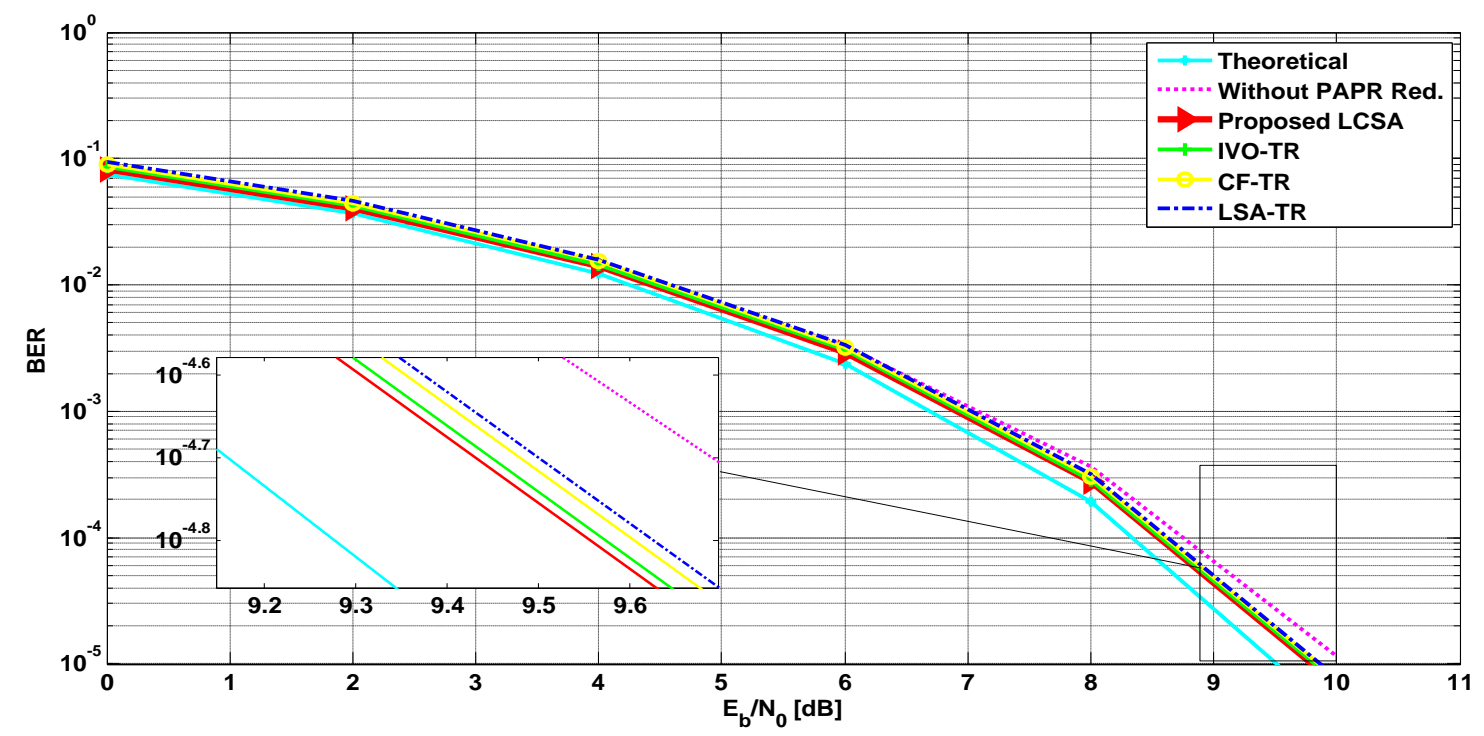

Fig.7. BER performance of different methods over AWGN channel

Table 5. Required $\boldsymbol{E}_{\boldsymbol{b}} / \boldsymbol{N}_{\boldsymbol{o}}$ for the different methods at BER $=\mathbf{1 0}^{\mathbf{- 5}}$

\begin{tabular}{|c|c|c|c|c|}
\hline Algorithm & LCSA & IVO-TR & CF-TR & LSA-TR \\
\hline $\boldsymbol{E}_{\boldsymbol{b}} / \boldsymbol{N}_{\boldsymbol{o}}$ at BER $=\mathbf{1 0}^{-\mathbf{5}}$ & 9.81 & 9.83 & 9.86 & 9.88 \\
\hline
\end{tabular}


DOI: https://dx.doi.org/10.26808/rs.ca.i10v5.03 International Journal of Computer Application (2250-1797) Issue 10 Volume 5, September- October 2020

\section{CONCLUSION}

In this paper, we have proposed a new PAPR reduction method for OFDM systems, which we refer to as a low-complexity signal addition (LCSA) method. The method designs a peak-reducing signal in the time-domain and then appends a few samples of the signal to the transmit signal instead of using the traditional way of reserving data subcarriers as done in tone reservation methods. Because of the design approach of the transmitted waveform, the method has a lower data rate loss than the tone reservation methods.

An investigation into the ability of the proposed method to reduce PAPR showed that it could achieve significant PAPR reductions with very low data rate losses while practically maintaining the average power of OFDM signals. The method also has a low runtime computational complexity of $O(M)$ where $M$ is far much less than $N$, the total number of subcarriers, in an OFDM system.

In comparison with IVO-TR, CF-TR and LSA-TR, the proposed method outperforms the three in terms of PAPR reduction, computational complexity, average transmit power increase, data rate loss and BER performances.

\section{REFERENCES}

[1] Y. Rahmatallah and S. Mohan, "Peak-to-average power ratio reduction in OFDM systems: A survey and taxonomy," IEEE Comm. Surveys and Tutorials, Vol. 15, No. 4, 2013, pp. 1567-1592.

[2] Y. Louët and J. Palicot, "A classification of methods for efficient power amplification of signals,"Annals of Telecom., vol. 63, No. 7-8, 2008, pp. 351-368.

[3] S. Takizawa and H. Ochiai, "PAPR Reduction of OFDM With Trellis Shaping Based on p -Norm Minimization," in IEEE Wireless Comm. Letters, vol. 8, no. 4, pp. 988-991, Aug. 2019.

[4] Tang, K. Qin, and H. Mei “A Hybrid Approach to Reduce the PAPR of OFDM Signals Using Clipping and Companding,"IEEE Access, Vol. 8, 2020, pp. 18984-18994.

[5] S. P. Valluri, V. Kishore, and V. M. Vakamulla,"A New Selective Mapping Scheme for Visible Light Systems,"IEEE Access, Vol. 8, 2020, pp. 18087-18096.

[6] Jawhar, L. Audah, M. Taher, K. Ramli, N. Shah, M. Musa, and M. Ahmed, "A Review of Partial Transmit Sequence for PAPR Reduction in the OFDM Systems,"IEEE Access, Vol. 7, 2019, pp. 18021-18041.

[7] H. Boche and U. J. Mönich, "Peak-to-average power control via tone reservation in general orthonormal transmission systems," IEEE Trans Signal Process, vol. 66, no. 13, 2018, pp. 3520-3528.

[8] J. Tellado, Peak to average power reduction for multicarrier modulation,Ph.D. dissertation, Dept. Elect. Eng., Stanford University, Stanford, CA, USA, 2000.

[9] T. Jiang, C. Ni, C. Xu, and Q. Qi, "Curve fitting based tone reservation method with low complexity for PAPR reduction in OFDM systems,"'IEEE Comm. Letters, vol. 18, No. 5, pp. 805-808,May 2014.

[10] H. Li, T. Jiang and Y. Zhou, "An Improved Tone Reservation Scheme With Fast Convergence for PAPR Reduction in OFDM Systems," IEEE Trans. on Broadcast., vol. 57, No. 4, pp. 902-906, Dec. 2011.

[11] H. Li, J. Wei, and N. Jin, "Low-Complexity Tone Reservation Scheme Using PreGenerated Peak-Canceling Signals,"IEEE Comm. Letters, Vol. 23 , Issue: 9, Sept. 2019, pp. 1586-1589.

[12] M. Sharif, M. Gharavi-Alkhansari, and B. H. Khalaj, "New Results on the Peak Power of OFDM Signals Based on Oversampling,"in Proc. IEEE ICC, Vol. 2, 2002, pp. 866-871. 
DOI: https://dx.doi.org/10.26808/rs.ca.i10v5.03 International Journal of Computer Application (2250-1797) Issue 10 Volume 5, September- October 2020

[13] Y. Cho, J. Kim, W. Yang, and C. Kang, MIMO-OFDM Wireless Communications with MATLAB, John Wiley \& Sons (Asia) Pte Ltd, Singapore, 2010.

[14] S. Boyd and L. Vandenberghe, Convex Optimization, Cambridge University Press, New York, USA, 2004.

[15] S. Kiambi, E. Mwangi, and G. Kamucha, "An Iterative Re-Weighted Least-Squares Tone Reservation Method for PAPR Reduction in OFDM Systems," WSEAS Trans. on Comm., ISSN / E-ISSN: 1109-2742 / 2224-2864, Vol. 18, 2019, pp. 153-161.

[16] H. J. Yin, R. Z. Yang, X. L. Luo, L. Jiang, and L. J. Zhu, Weighted tone reservation for OFDM PAPR reduction, U.S. Patent 7796498, 2008.

[17] L. Xin and W. Yi, A New Weighted Tone Reservation Method for PAPR Reduction in OFDM Systems, Journal of Communication, Vol. 9, No. 12, 2014, pp. 980-986.

[18] J. Song and H. Ochiai, "Performance analysis for OFDM signals with peak cancellation," IEEE Trans. on Comm., Vol. 64, No. 1, 2016, pp. 261-270.

[19] A. Gatherer and M. Polley, "Controlling clipping probability in DMT transmission," in Proc. 31st Asilomar Conf. Signals, Syst., Comput., Vol. 1, Nov. 1997, pp. 578-584. 